\title{
A shell model representation with antibound states
}

\author{
R. Id Betan ${ }^{a, b}$, R.J. Liotta ${ }^{a}$, N. Sandulescu ${ }^{a, c}$, T. Vertse ${ }^{a, d}$ \\ a Royal Institute of Technology, AlbaNova University Center, SE-10691 Stockholm, Sweden \\ ${ }^{\mathrm{b}}$ Departamento de Fisica, FCEIA, UNR, Avenida Pellegrini 250, 2000 Rosario, Argentina \\ ${ }^{\mathrm{c}}$ Institute of Physics and Nuclear Engineering, P.O. Box MG-6, Bucharest-Magurele, Romania \\ ${ }^{\mathrm{d}}$ Institute of Nuclear Research of the Hungarian Academy of Sciences, Pf. 51, H-4001 Debrecen, Hungary \\ Received 28 November 2003; received in revised form 13 January 2004; accepted 15 January 2004
}

Editor: J.-P. Blaizot

\begin{abstract}
An unified shell model scheme is introduced that evaluates simultaneously the contributions of bound single-particle states, Gamow resonances and antibound states to processes occurring in the continuum part of nuclear spectra. This new scheme allows us to study the effect of the antibound pole and the remaining part of the complex continuum separately. The calculations, performed in the complex energy plane, are applied to the study of weakly bound nuclei. The influence of antibound states upon physical quantities in light as well as in heavy nuclei is assessed.
\end{abstract}

(c) 2004 Elsevier B.V. All rights reserved.

PACS: 25.70.Ef; 23.50.+z; 25.60.+v; 21.60.Cs

\section{Introduction}

One major difficulty related to the microscopical description of nuclei close to the drip lines is the treatment of the continuum coupling. The first attempts to introduce the continuum coupling into shell model calculations were done more than forty year ago in the framework of the continuum shell model (CSM) [1]. In CSM calculations one considers usually at most one particle scattered into the continuum part of the single-particle spectrum. For more complicated configurations, with two or more particles moving in the continuum, the CSM calculations become unfeasible

\footnotetext{
E-mail address: idbetan@ nuclear.kth.se (R. Id Betan).
}

due to the extremely large dimensions of the shell model basis. The size of the CSM basis can be eventually reduced to manageable dimensions if one could restrict the calculations to that continuum configurations based on single-particle resonant states, which carry the most important contribution of the continuum coupling. However, in positive energy representations, as the one used in the CSM, there is not a unique wave function associated to a given singleparticle resonance. Such unique (discrete) wave functions, the so-called Gamow functions [2], can be defined only in the complex energy plane. They correspond to the outgoing solutions of the one-body Schrödinger equation [3]. The Gamow states, together with a set of scattering states belonging to a contour in the complex energy plane form a complete repre- 
sentation, the Berggren representation [4]. Based on this representation a shell model in the complex energy plane (CXSM) was recently presented [5-8].

In its initial form the CXSM was formulated starting from a Berggren representation consisting of bound states, Gamow resonances and complex scattering states. In this Letter we will further extend the CXSM including also the antibound states in the single-particle basis. That is, the antibound states will be part of the single-particle representation in the same fashion as bound states are in standard shell model calculations. This new formalism allows us to analyze the role of the antibound state separately from the remaining part of the (complex) continuum. Within this representation we will investigate, from the shell model perspective, the effect of the antibound states on the structure of exotic nuclei, particularly the ones which form neutron halos. But we want to stress that it is not the intention of this work to provide a general theory of nuclear halos but rather to show how to include the antibound states in the CXSM and the influence that antibound states may have upon nuclear properties.

\section{Formalism}

The shell model in the complex energy plane is based on the Berggren representation. Although this representation and its use in shell model type calculations has been described before [4-10], we will briefly present it here again for clarity of presentation.

The Berggren representation is a complete basis formed by a discrete set of wave functions corresponding to the poles of the S-matrix, plus a set of scattering states with energies belonging to a continuous path in the complex energy plane. In this representation the completeness relation can be written as [4]

$$
\begin{aligned}
\delta\left(r-r^{\prime}\right)= & \sum_{n} w_{n}(r) w_{n}\left(r^{\prime}\right) \\
& +\int_{L^{+}} d E u(r, E) u\left(r^{\prime}, E\right) .
\end{aligned}
$$

The summation in the expression above runs over all the bound states and over those poles of the S-matrix which are enclosed by the real energy axis and the contour $L^{+}$.
An important feature of the Berggren representation is that the scalar product is defined as the integral of the wave function times itself, and not its complex conjugate. Therefore, the related metric, i.e., the Berggren metric, is not Hermitian. As a result, the matrix elements corresponding to any operator between the vectors of the Berggren basis may become complex numbers. However, observable (physical) quantities remain real if all basis vectors are included in the Berggren representation.

The contour in the complex energy plane can have in principle any form [10]. Nevertheless, as shown in Refs. [5,6] for the case of two-particle systems, a particular class of rectangular contours is much more appropriate for CXSM calculations. The reason is that such contours can generate in the two-particle complex energy plane a region free of two-particle uncorrelated states. This property is very important both for the identification and the calculation of twoparticle resonant states [6].

As an illustration, we show in Fig. 1 a rectangular contour in the complex energy plane that encloses three Gamow resonances. The contour is defined by the vertices $V_{i}$ and the energies of the Gamow resonances are given by the points $G_{i}$. In the case of the figure the contour finishes at the point $V_{5}$. This cut-off of high lying states is a common feature of the shell model, where only a limited number of shells are included.

In order to treat numerically the scattering states one discretizes the contour integral of Eq. (1) such that [9]

$\int_{L^{+}} d E u(r, E) u\left(r^{\prime}, E\right)=\sum_{p} h_{p} u\left(r, E_{p}\right) u\left(r^{\prime}, E_{p}\right)$,

where $E_{p}$ and $h_{p}$ are defined by the procedure one uses to perform the integration. In the Gaussian method $E_{p}$ are the Gaussian points and $h_{p}$ the corresponding weights. Therefore, the orthonormal basis vectors are given by the set of bound and Gamow states, i.e., $\left\langle r \mid \varphi_{n}\right\rangle=\left\{w_{n}\left(r, E_{n}\right)\right\}$ and the discretized scattering states, i.e., $\left\langle r \mid \varphi_{p}\right\rangle=\left\{\sqrt{h_{p}} u\left(r, E_{p}\right)\right\}$. This defines the Berggren representation used in the CXSM calculations.

As in any standard shell model, in CXSM the multiparticle basis states are formed by the tensorial product of the ordered single-particle states belonging to 


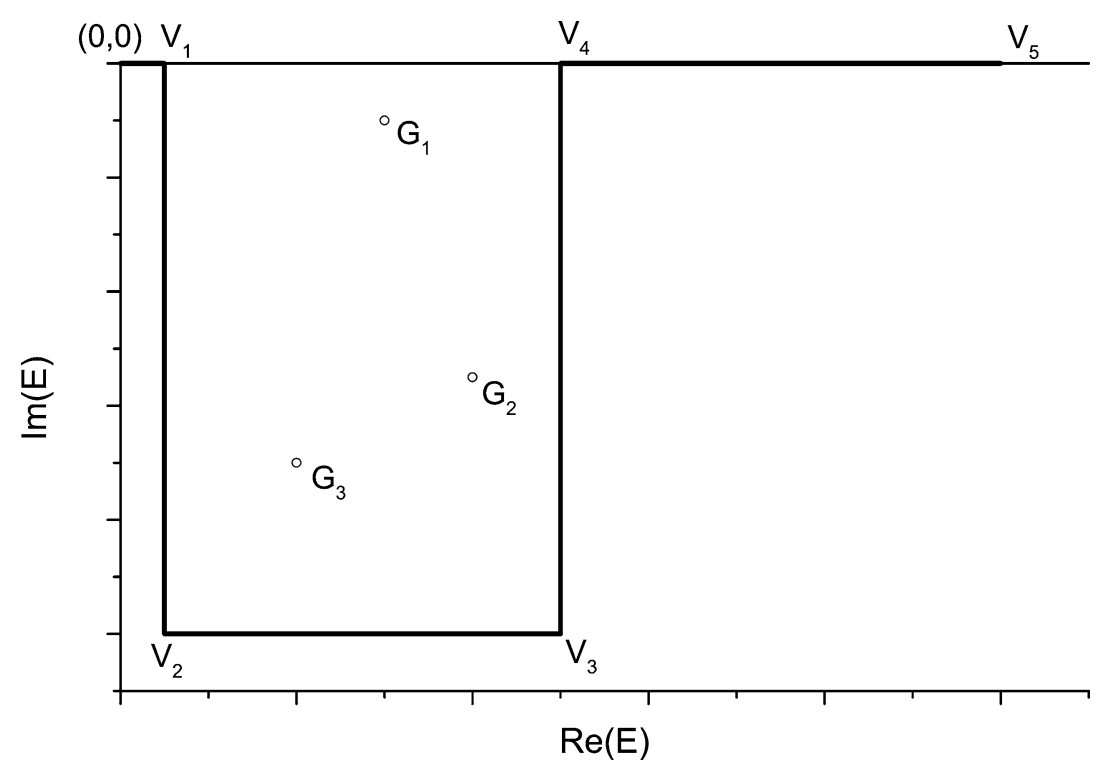

Fig. 1. One-particle complex energy plane. The broad line indicates the contour. The points $V_{i}$ are the vertices defining the contour. The open circles labelled by $G_{i}$ indicate the complex energy of the Gamow resonances enclosed by the contour.

the chosen Berggren representation. The matrix elements of the residual interaction are calculated within this representation by using the Berggren metric. Thus for a separable interaction the matrix elements have the form:

$$
\langle\widetilde{k l} ; \alpha|V| i j ; \alpha\rangle=-G_{\alpha} f_{\alpha}(k l) f_{\alpha}(i j),
$$

where $G_{\alpha}$ is the strength of the force. One can see that due to the Berggren metric on the r.h.s. appears the form factor $f_{\alpha}(k l)$ and not $f_{\alpha}(k l)^{*}$ as in the standard Hilbert metric. Consequently, the standard dispersion relation for a two-particle system corresponding to a separable force becomes $[5,6]$

$-\frac{1}{G_{\alpha}}=\sum_{i \leqslant j} \frac{f_{\alpha}^{2}(i j)}{\omega_{\alpha}-\epsilon_{i}-\epsilon_{j}}$,

where $\omega_{\alpha}$ are the correlated energies. One notices again that due to the Berggren metric in the dispersion relation appears the square of the form factors $f_{\alpha}$ and not the square of its absolute value. For more details see Ref. [6].

In the CXSM calculations done until now the only complex energy poles considered in the Berggren representation are the Gamow resonances. The new elements that we will include in this Letter are the antibound states (they are also known in the literature as virtual states).

The antibound states are the outgoing solutions of the Schrödinger equation with negative imaginary wave numbers, i.e., $k=-i|k|$. Thus the energy corresponding to an antibound state is real and negative, as for the bound states, but the tail of the corresponding wave function diverges exponentially at large distances.

An antibound state close to threshold manifests itself on the real energy axis through the localization properties of the low-lying scattering states. This can be shown [11] by considering a mean field that has an antibound $s$-state with energy $E_{0}\left(k_{0}=-i\left|k_{0}\right|\right)$ lying near threshold. One thus finds that the radial scattering wave function with energies $E=\hbar^{2} k^{2} / 2 \mu$ ( $k$ real and positive) close to zero can be approximated inside the mean field region by

$\mathcal{R}_{l}(k r) \approx \sqrt{\frac{2 k\left|k_{0}\right| a}{k^{2}+\left|k_{0}\right|^{2}}} \mathcal{R}_{l}\left(\left|k_{0}\right| r\right)$,

where $a$ is a constant depending on the normalization chosen for the wave function $\mathcal{R}_{l}\left(\left|k_{0}\right| r\right)$. This expression shows that close to threshold the radial dependence of the scattering wave functions inside the mean field region depends upon the energy only through the 


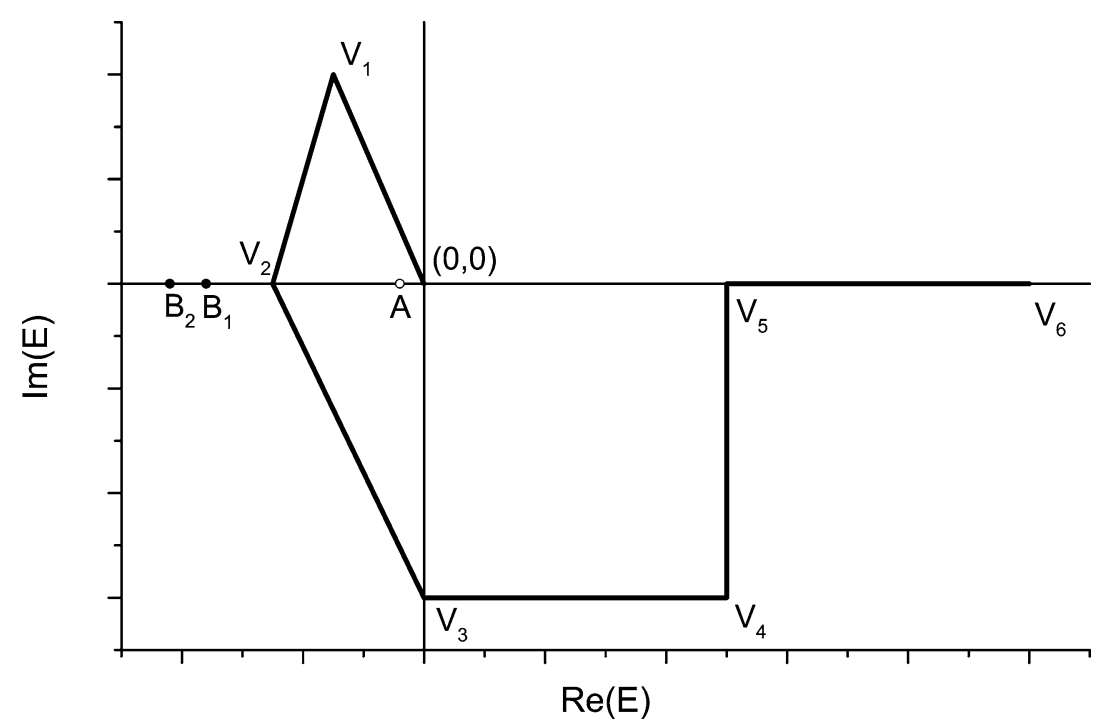

Fig. 2. One-particle complex energy plane. The broad line indicates the contour embracing the antibound state A, indicated by an open circle. The points $\mathrm{V}_{i}$ are the vertices defining the contour. The points $\mathrm{B}_{i}$ indicate bound states.

square root factor. This factor is maximum at $k=\left|k_{0}\right|$. Therefore, in an energy interval located around $\left|E_{0}\right|$ the scattering states have an increased localization. These scattering states will represent indirectly the effect of the antibound state in any type of continuum shell model calculations based on real energy representations.

In the framework of the CXSM one has the possibility to include the antibount states directly in the Berggren basis and treat them formally on the same footing as the bound states and Gamow resonances. This can be done by choosing a contour as the one shown in Fig. 2. In this figure the points $V_{i}$ define the contour while the open circle $A$ corresponds to the energy of the antibound state.

In the Berggren representation to each partial wave corresponds a contour. These contours may be different to each other. In the extended CXSM calculations presented in the next section the Gamow resonances and the antibound poles will be enclosed by the contours shown in Figs. 1 and 2.

\section{Applications}

To show the convenience of the formalism presented above we will apply it to cases where antibound states are known to be important. This is particularly the case of halo type nuclei. In what follows we will first consider the typical case of ${ }^{11} \mathrm{Li}$.

The existence of a low-lying virtual $s$-state in ${ }^{10} \mathrm{Li}$ has important consequences for the correlations developed in ${ }^{11} \mathrm{Li}$ [12]. As discussed above, an antibound state close to the continuum threshold enhances the localization of the low-lying scattering states. Therefore, the $s$-wave content of the ground state of ${ }^{11} \mathrm{Li}$ is also increased, reaching the corresponding (large) experimental value. Moreover, the antibound state in ${ }^{10} \mathrm{Li}$ can affect the excited spectrum of ${ }^{11} \mathrm{Li}$ as well as the ground state. These effects of the antibound states will be studied here from the viewpoint of the CXSM.

It is by now well-known that in the description of ${ }^{11} \mathrm{Li}$ the two relevant single-particle states, as specified by the experimental spectrum of ${ }^{10} \mathrm{Li}$, consist of a low-lying antibound (or virtual) $s_{1 / 2}$ state at about $-50 \mathrm{keV}$, and a $p_{1 / 2}$-resonance at about $540 \mathrm{keV} \mathrm{[13].}$ A $p$ resonance at around $0.250 \mathrm{MeV}$ is also considered in Ref. [14]. The two-body correlations induce a bound ground state in ${ }^{11} \mathrm{Li}$ at about $-0.295 \mathrm{MeV}$. Notice that we are here using the shell-model language, where the core $\left({ }^{9} \mathrm{Li}\right)$ is considered as inert, the singleparticle states are given by ${ }^{10} \mathrm{Li}$ and the two-body nucleus is ${ }^{11} \mathrm{Li}$ [15]. 
In the first step of the CXSM one evaluates the single-particle states of the unbound nucleus ${ }^{10} \mathrm{Li}$. As in Ref. [16,17], for the central field we choose a Woods-Saxon potential with different depths for even and odd orbital angular momenta $l$. One thus simulate the effect of core polarisation upon singleparticle states $[16,18]$. In order to study the influence of the $p_{1 / 2}$ resonance upon the halo properties, we will perform two calculations corresponding to the energies $200 \mathrm{keV}$ and $500 \mathrm{keV}$ of the $0 p_{1 / 2}$ resonant state. We will start studying the $200 \mathrm{keV}$ case and afterwards the $500 \mathrm{keV}$ case will be presented. At the end we will compare the results and discuss the similarities and differences between the two cases.

For the $200 \mathrm{keV}$ case we use the Woods-Saxon potential given by $a=0.67 \mathrm{fm}, r_{0}=1.27 \mathrm{fm}, V_{0}=$ 50 (36.9) $\mathrm{MeV}$ and $V_{\text {so }}=16.5$ (12.624) $\mathrm{MeV}$ for $l$ even (odd). With these parameters we found the single-particle bound states $0 s_{1 / 2}$ at $-23.278 \mathrm{MeV}$ and $0 p_{3 / 2}$ at $-2.589 \mathrm{MeV}$ forming the ${ }^{9} \mathrm{Li}$ core. The valence poles are the low lying resonances $0 p_{1 / 2}$ at $(0.195,-0.047) \mathrm{MeV}$ and $0 d_{5 / 2}$ at $(2.731,-0.545)$ $\mathrm{MeV}$ and the wide resonance $0 d_{3 / 2}$ at $(6.458,-5.003)$ MeV. Besides, the state $1 s_{1 / 2}$ appears as an antibound state $^{1}$ at $-0.050 \mathrm{MeV}$. We thus reproduce the experimental single-particle energies giving from the very beginning unequivocal endorsement to the low lying $s$-state as due to an antibound state.

We also found other resonances at high energies. However, we include in the basis single-particle states lying up to $10 \mathrm{MeV}$ of excitation energy only. We found that expanding the basis from this limit does not produce any effect upon the calculation up to the six digits of precision that we require.

The next step in the calculation is to adopt a residual interaction. We will use a separable force and, therefore, the Hamiltonian matrix reduces to the dispersion relation give by Eq. (4). For the field in the separable interaction we use the form:

$f(r)=\frac{f_{0}}{1+\exp \left(\left(r-R^{\prime}\right) / a^{\prime}\right)}$,

\footnotetext{
1 The principal quantum number $n$ labelling the single-particle states indicates that the corresponding wave functions are localized in a region inside the nucleus and that its real part has in that region $n$ nodes, excluding the origin.
}

where $f_{0}=35, R^{\prime}=18 \mathrm{fm}$ and $a^{\prime}=1.3 \mathrm{fm}$. To evaluate the ground state of ${ }^{11} \mathrm{Li}$ we adjust the strength $G$ of the separable interaction to reproduce the corresponding energy, i.e., $-295 \mathrm{keV}$. We thus obtained $G=0.00194 \mathrm{MeV}$.

With the mean field and the two-body interaction thus established we evaluated the ground state wave function. First, we performed the calculations by choosing the real energy as a contour. In this case the wave function is spread over many components. The largest of these components corresponds to configurations $p_{1 / 2} \otimes p_{1 / 2}$ lying close to $400 \mathrm{keV}$ (i.e., about twice the energy of the $0 p_{1 / 2}$ resonance) and $s_{1 / 2} \otimes s_{1 / 2}$ lying close to threshold (i.e., close to twice the energy of the antibound state). The wave function consists of $44 \% s$-states, $48 \% p$-states and $8 \%$ $d$-states, as expected [13,19].

If we keep the strength constant at $G=0.00194$ $\mathrm{MeV}$ and leave out either the antibound pole or the $l=0$ contour encircling the pole, the $l=0$ content of the wave function increases to $77 \%$. If, however, we readjust the value of $G$ in order to get the state at the correct position at $-295 \mathrm{keV}$ then the $l=0$ content of the wave function increases further up to $98 \%$. This shows that the antibound pole and the scattering states along the $l=0$ complex path are adding up with very strong destructive interference and this reduces the $l=0$ content of the wave function somewhat below the $l=1$ content.

A remarkable feature of the calculation is that the antibound state exerts such a strong effect upon the two-particle wavefunction. From a CXSM point of view this is because the energy corresponding to the configuration $\left(1 s_{1 / 2}\right)^{2}$ is very close (in the complex energy plane) to the two-particle energy. But it can also be understood from a continuum shell-model point of view. As discussed in the previous section, an antibound pole close to the continuum threshold induces a large localization of the low-lying scattering states inside the nucleus. This feature can be seen in Fig. 3, where we give the localization of the scattering states, $L(E)$, defined as

$L(E)=\int_{0}^{1.2 R_{N}} \mathcal{R}_{l=0}^{2}(k r) r^{2} d r$,

where $R_{N}=2.6 \mathrm{fm}$ is the core nuclear radius. As seen in Fig. 3, there is a strong increase of the localization 


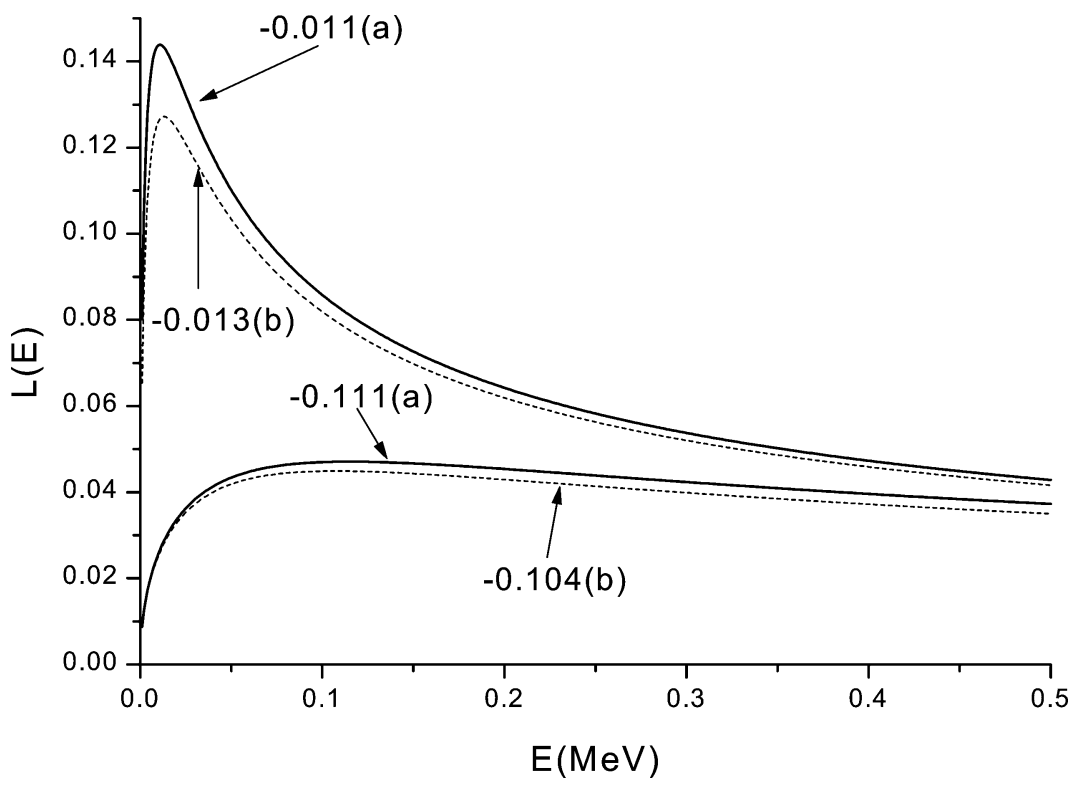

Fig. 3. Localization $L(E)$, Eq. (6), in the presence of low-lying antibound (a) and bound (b) $s$-states. The numbers labeling the curves are the energies of the poles in MeV.

as the energies of the poles approaches threshold. This property is also responsible for a similar increase in the elastic cross section (see Figs. 12 and 13 of Ref. [20]). From Fig. 3 one notices also that the localization of the scattering states does not depend on whether the S-matrix pole $E_{0}$ corresponds to a bound or to an antibound state. This strong localization of the scattering states generates, through the matrix elements of the residual interaction, large components of the $s$-wave in the two-body wave function. This property shows that the nuclear halo should not be traced back to the appearance of an antibound $s$-state close to threshold since weakly bound $s$-state would give a similar effect. That is, halos may be induced by a strong pairing interaction in the presence of antibound $s$-states close to threshold or weak pairing interaction in the presence of weakly bound $s$-states. We would like to stress again that in this work we propose a theoretical framework to treat the first option correctly and not to provide a general theory of nuclear halos.

So far we have shown the advantages of the CXSM to evaluate the effect of the antibound state on already known properties of the ground state of the halo nucleus ${ }^{11} \mathrm{Li}$. However, the transparency of the method becomes essential in the search for other physically meaningful two-particle states in the continuum. In what follows we discuss the problem of low-lying $0^{+}$ excitations and their influence upon the neutron halo.

Within the CXSM two-particle resonances are easy to calculate since they appear as a result of the diagonalization of the Hamiltonian (which in our case reduces to the solution of the dispersion relation) in the complex energy plane. Thus we found that the first excited state (i.e., the state $0_{2}^{+}$) appears at the complex energy $(0.202,-0.137) \mathrm{MeV}$. The corresponding wave function consists of nearly $100 \%$ $p$-states, with a small admixture of $s$-states.

It is interesting to analyze how this state is built up by the two-body interaction starting from the zerothorder configuration $\left(0 p_{1 / 2}\right)^{2}$. For this we increased the interaction gradually starting from $G=0$, as seen in Fig. 4. As the attractive interaction increases the resonance becomes narrower and approaches threshold, as expected from perturbation theory. However, a point is reached where continuum configurations become important and the resonance widens. This happens at $G=0.0005 \mathrm{MeV}$ in the figure. Up to this point the resonance is a purely $\left(0 p_{1 / 2}\right)^{2}$ state and, therefore, it is localized inside the nucleus. That is, it is a physically meaningful resonance. But from here on other configurations become important. These configurations 


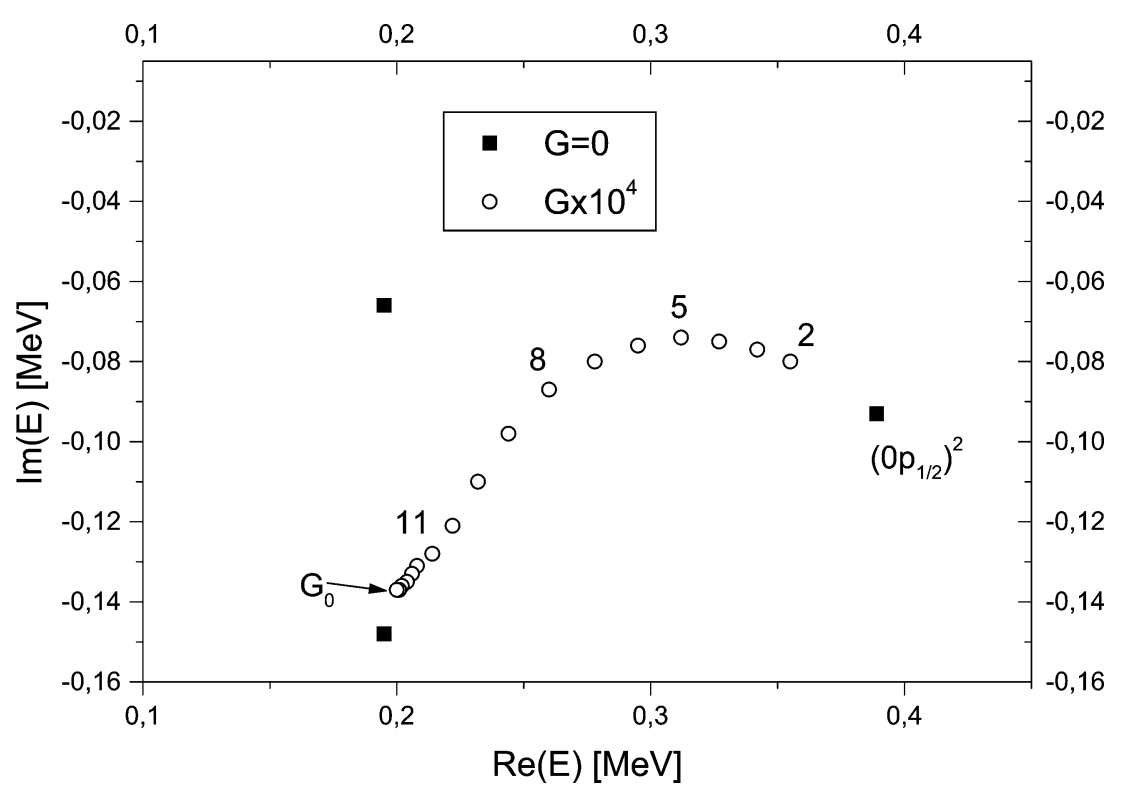

Fig. 4. Evolution of the energies of the two-particle resonance ${ }^{11} \mathrm{Li}\left(0_{2}^{+}\right)$as a function of $G$ (in $\mathrm{MeV}$ ) for the $200 \mathrm{keV}$ case. The numbers labeling the open circles are the values of $G \times 10^{4} \mathrm{MeV}$. The strength adjusted to obtain the ground state is $G_{0}=19.4 \times 10^{-4} \mathrm{MeV}$.

are overwhelmingly those where one of the particles moves in the continuum and the other in the resonance $0 p_{1 / 2}$. More specifically, in the two-particle resonance that we are studying the most important states in the continuum are those corresponding to $p_{1 / 2}$ waves with energies corresponding to the points on the segment $V_{1}-V_{2}$ in Fig. 1 . At $G=G_{0}=0.00194 \mathrm{MeV}$, corresponding to the $G$-value fitting the ground state, there is a strong mixing with the continuum configurations. Increasing $G$ farther the configuration $\left(0 p_{1 / 2}\right)^{2}$ looses its importance, the resonance is split in a number of pieces and eventually dissolves into the continuum. However, since these configurations virtually include only $p$-waves the wavefunctions still consist of only $p$-states.

The analysis that we have done so far is based upon the assumption that the $p$ resonance in ${ }^{10} \mathrm{Li}$ is located at $200 \mathrm{keV}$. To see the influence of this resonance on the structure of the halo we will now analyze the ground and the excited states of ${ }^{11} \mathrm{Li}$ by using the $500 \mathrm{keV}$ case. For this we adopted the Woods-Saxon depth $V_{0}=35.366 \mathrm{MeV}$ for odd $l$-values, keeping all other parameters as before. We thus obtained the energy $(0.470,-0.197) \mathrm{MeV}$ for the state $0 p_{1 / 2}$. The state $0 p_{3 / 2}$, belonging to the core, is found now at $-2.016 \mathrm{MeV}$. The other odd $l$-value poles lie beyond the range of energies included here. But, nevertheless, we have checked that they do not affect the results.

We kept the two-particle interaction used in the previous case, except that now $R^{\prime}=7.9 \mathrm{fm}$ and the strength necessary to adjust the energy of ${ }^{11} \mathrm{Li}(\mathrm{gs})$ is $G=0.00694 \mathrm{MeV}$.

As before, we found that on the real energy axis the ground state wave function is spread in many components. The largest of them lie close to threshold for the configurations $s_{1 / 2} \otimes s_{1 / 2}$ and around $1 \mathrm{MeV}$ for the $p_{1 / 2} \otimes p_{1 / 2}$ configurations. The wave function consists of $49 \% s$-states, $39 \% p$-states and $12 \%$ $d$-states, which is also within the range of accepted values $[13,21]$.

Since the position of the $p_{1 / 2}$ pole seems likely to correspond to the present $500 \mathrm{keV}$ case [21], we will analyze here the effects of the antibound and the Gamow poles upon the ground state of ${ }^{11} \mathrm{Li}$ by using the contours of Figs. 1 and 2. We therefore present in Table 1 the contribution of different configurations to that ground state. The corresponding complex amplitudes depend on the chosen contours and have no direct physical meaning. But the total content of a given partial wave in the bound ground state wave function, which is a physical quantity, does not depend upon the chosen contour. From Table 1 we can see 
Table 1

The contribution of partial wave configurations $\left(s_{1 / 2}\right)^{2},\left(p_{1 / 2}\right)^{2}$ and $\left(d_{5 / 2}\right)^{2}$ to the ground state wave function calculated in the complex energy plane. For each partial wave are given the square amplitude of the pole-pole term and the sum of the square amplitudes corresponding to the pole-scattering and scattering-scattering terms. The total contribution of each partial wave is given in the last line

\begin{tabular}{lccc}
\hline & $\left(s_{1 / 2}\right)^{2}$ & $\left(p_{1 / 2}\right)^{2}$ & $\left(d_{5 / 2}\right)^{2}$ \\
\hline Pole-pole & $(12.936,-0.039)$ & $(0.642,-0.204)$ & $(0.127,0.011)$ \\
Pole-scat. & $(-29.365,0.079)$ & $(-0.279,0.221)$ & $(-0.031,-0.018)$ \\
Scat.-scat. & $(16.921,-0.040)$ & $(0.022,-0.017)$ & $(-0.002,0.007)$ \\
Total & $(0.492,0.0)$ & $(0.385,0.0)$ & $(0.094,0.0)$ \\
\hline
\end{tabular}

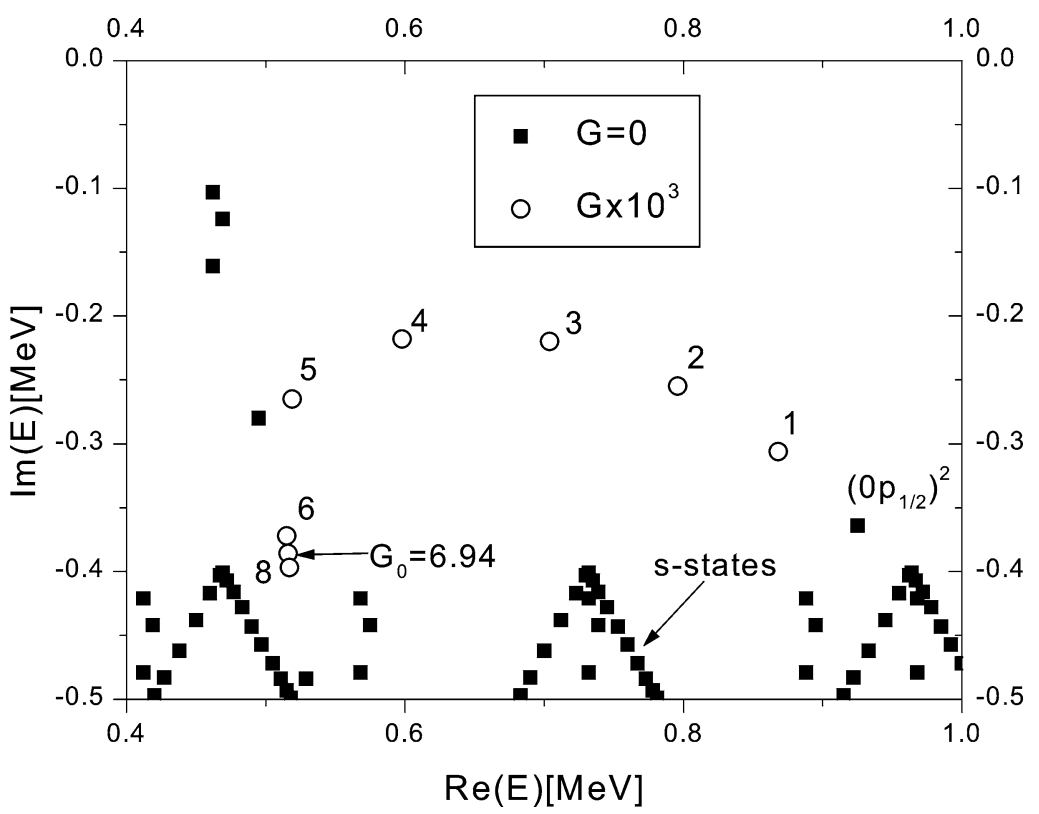

Fig. 5. As Fig. 4 for the $500 \mathrm{keV}$ case except that the numbers are the values of $G \times 10^{3} \mathrm{MeV}$ and $G_{0}=6.94 \times 10^{-3} \mathrm{MeV}$.

that for the $p$ and $d$ waves the configurations are built mainly on the corresponding Gamow resonances. The situation is different for the $s$-wave since apart from the configurations built upon the antibound state there is also an important contribution coming from the complex scattering states. This contribution is given mainly by those $s$ scattering states located on the segments $(0,0)-V_{1}$ and $V_{1}-V_{2}$ of Fig. 2 , which are the closest to the antibound state.

Up to this point there is not much difference between the 200 and the $500 \mathrm{keV}$ cases, which may explain why various studies of the halo structure of ${ }^{11} \mathrm{Li}(\mathrm{gs})$ with the common feature of having lowlying $s$ - and $p$-states, provide similar results [21]. This is because the wave function of ${ }^{11} \mathrm{Li}(\mathrm{gs})$ is mainly controlled by low-spin single-particle states lying close to the continuum threshold. The exact positions of the resonances do not influence the wave function very much. However, the position of the singleparticle poles may have a fundamental importance to determine the physically meaningful excited states. The states arising from the particles moving in the continuum are not localized inside the nucleus and, therefore, will be weakly affected or not affected at all by the interaction. This can be seen in Fig. 5, where we present the evolution of the state $0_{2}^{+}$as a function of the strength $G$. The $0 p_{1 / 2}$ resonance is now wider and higher in energy than before. As a result, the point corresponding to $G=0$ in the figure is closer to points coming from the continuum contour. Yet, these continuum states do not seem to affect the resonance as $G$ increases. That is, the behaviour of 
Table 2

Square of the wave function amplitude $X\left((n l j)^{2}\right)$, where $(n l j)$ indicates the quantum number of the single-particle resonance, and the sum $S(l)$ corresponding to the physical resonance of Fig. 5. The values of $G$ are in $\mathrm{MeV}$ and $G_{0}=0.00694$

\begin{tabular}{|c|c|c|c|c|}
\hline$G$ & $X^{2}\left(\left(1 s_{1 / 2}\right)^{2}\right)$ & $S(0)$ & $X^{2}\left(\left(0 p_{1 / 2}\right)^{2}\right)$ & $S(1)$ \\
\hline 0.001 & $(0.00,0.00)$ & $(-0.00,0.00)$ & $(1.00,0.00)$ & $(0.00,-0.00)$ \\
\hline 0.003 & $(0.05,0.02)$ & $(-0.10,0.10)$ & $(1.06,0.06)$ & $(0.00,-0.05)$ \\
\hline 0.005 & $(-0.01,0.11)$ & $(-0.25,-0.12)$ & $(0.59,0.30)$ & $(0.47,-0.19)$ \\
\hline$G_{0}$ & $(-0.04,0.02)$ & $(-0.00,-0.11)$ & $(0.23,0.18)$ & $(0.74,-0.12)$ \\
\hline 0.008 & $(-0.03,0.01)$ & $(0.01,-0.08)$ & $(0.18,0.14)$ & $(0.79,-0.10)$ \\
\hline
\end{tabular}

the resonance as $G$ is varied is very similar to that in Fig. 4 as well as to resonances in non-halo nuclei [6]. The reason for this is that those points, label " $s$-states" in the figure, correspond to configurations of the type $c s_{1 / 2} 1 s_{1 / 2}$, where " $c$ " labels points in the segments $(0,0)-V_{1}$ and $V_{1}-V_{2}$ of Fig. 2. The overlap between these configurations and the mainly $\left(0 p_{1 / 2}\right)^{2}$ configuration of the resonance is small. Only at large values of $G$ (above $G=0.004 \mathrm{MeV}$ in the figure), the resonance starts to feel the presence of the continuum states. The remarkable feature of the figure is the sudden turning down of the curve corresponding to the physical resonance at $G=0.005 \mathrm{MeV}$. As $G$ increases in this region the continuum plays a mounting role. As in the $200 \mathrm{keV}$ case above, the most important of the continuum configurations are those in which one particle moves in the continuum and the other in the $0 p_{1 / 2}$ resonance. There could be many comparatively large configurations of these type and it would not be useful to give all of them. More instructive is to show their contribution to the normalization of the wave function in this case where, in contrast to the ground state case of Table 1, the zeroth order energies are not very close to the energy of the state $0_{2}^{+}$. We thus define $S(l)$ as the sum of the squares of the amplitudes corresponding to configurations where at least one of the two particles moves in continuum states. On the real energy axis the sum of $S(l=1)$ and $X^{2}\left(\left(0 p_{1 / 2}\right)^{2}\right)$, where $X$ is the wave function amplitude, is the probability of the $p$-content of the wave function. This is a quantity that we evaluated above for the ground state. The dependence of these quantities upon $G$ corresponding to the state $0_{2}^{+}$is shown in Table 2. Since we are studying states with complex energies the numbers $S$ as well as $X^{2}$ are complex in this table. Moreover, their absolute values could be larger than 1 although the sum of all possible $l$-contributions is normalized to $(1,0)$. This is a good example of the non-Hermitian character of the Berggren metric.

One sees in this table that the two-particle resonance starts to mix with the continuum at $G=$ $0.005 \mathrm{MeV}$ and at $G=G_{0}$ it is composed mainly of continuum configurations. Therefore, at this point it has already lost its localization features. It has become a part of the continuum background.

We are now in a position to recognize other systems where halos may be present. We thus looked for nuclei which may be considered shell-model cores lying on the neutron drip line with low-lying single-particle resonances carrying low-spin. Following the trend of single-particle states in the relativistic mean field calculations we found that $Z=20, N=50$ may be such a core. In order to simulate the order of the single particle states given by the relativistic calculations we used a Woods-Saxon potential defined by $a=0.67 \mathrm{fm}$, $r_{0}=1.27 \mathrm{fm}, V_{0}=39 \mathrm{MeV}$ and $V_{s o}=22 \mathrm{MeV}$. With this potential the antibound $2 s_{1 / 2}$ state (note that $n=$ 2) appears again at $-0.050 \mathrm{MeV}$. But now the next valence shells are $1 d_{5 / 2}$ at $(0.469,-0.048) \mathrm{MeV}, 1 d_{3 / 2}$ at $(2.080,-1.525) \mathrm{MeV}, 0 g_{7 / 2}$ at $(6.739,-0.738)$ $\mathrm{MeV}$ and $0 h_{11 / 2}$ at $(5.344,-0.102) \mathrm{MeV}$. The states in the core are ordered as usual. As expected, the highest of these is the state $0 g_{9 / 2}$, lying at $-2.276 \mathrm{MeV}$. Using the same separable interaction as before and assuming again that the ground state of ${ }^{72} \mathrm{Ca}$ lies at $-295 \mathrm{keV}$, we obtained for the strength of the interaction the value $G_{0}=0.00174 \mathrm{MeV}$. Close to this $G_{0}$ value we found also a low-lying two-particle resonance with the energy of about $(0.550,-0.350) \mathrm{MeV}$. The behaviour of this $0_{2}^{+}$resonance as a function of $G$ is very similar to the $500 \mathrm{keV}$ case of Fig. 5. The discussion performed there is also valid here and, therefore, we will not analyzed this rather academic case farther. But it is important to point out that in this and the other cases presented here, we have been careful 
to choose contours that leave the region around the two-particle resonances in the complex energy plane free of continuum configurations. We thus established an "allowed" region [6]. Otherwise the two-particle resonance would be embedded in a see of continuum states, making the calculations difficult and the evaluated quantities unreliable.

\section{Summary}

In conclusion, we have presented in this Letter a new formalism to treat antibound states exactly and on the same footing as bound states and Gamow resonances. The antibound states and the Gamow resonances are selected by appropriate contours in the complex energy plane. Due to the complex singleparticle representation used in the present shell model formalism, the contribution of the pole-pole, polecontinuum and continuum-continuum configurations in the two-particle systems can be easily analyzed. The effects induced by antibound states and the continuum encircling the poles can be studied separately. The advantage of the formalism was illustrated for the halo type nuclei ${ }^{11} \mathrm{Li}$ and ${ }^{72} \mathrm{Ca}$. We confirm that antibound states lying close to the continuum threshold are of a fundamental importance to build up the halo. But we found that in the ground state of the ${ }^{11} \mathrm{Li}$ the large contribution of the antibound pole is partly cancelled by the complex continuum. We also found that an excited low-lying two-particle resonance may exist in these nuclei. For the case of ${ }^{11} \mathrm{Li}$ this low-lying resonant appears in the energy range of $0.2-0.5 \mathrm{MeV}$, which is the same as the one suggested in previous studies based on real energy representations [22-24]. However, the CXSM calculations exhibit a very drastic change in the structure of the resonant excited state when the strength of the force is approaching the value used for the determination of the ground state. This indicates that the excited state is strongly mixed with the continuum background. It is therefore rather difficult to conclude at this stage of the calculations whether this is a physical two-particle resonance or not. More detailed investigations of the behaviour of two-particle resonant states in halo type nuclei would require the application of the CXSM with non-separable residual forces commonly used in other models. This work is in progress.

\section{Acknowledgements}

This work has been supported by FOMEC and Fundación Antorchas (Argentina), by the Hungarian OTKA fund Nos. T37991 and T29003 and by the Swedish Foundation for International Cooperation in Research and Higher Education (STINT).

\section{References}

[1] A. Volya, V. Zelevinsky, Phys. Rev. C 67 (2003) 054322, and references therein.

[2] G.A. Gamow, Z. Phys. 51 (1928) 204.

[3] A.J.F. Siegert, Phys. Rev. 56 (1939) 750.

[4] T. Berggren, Nucl. Phys. A 109 (1968) 265.

[5] R. Id Betan, R.J. Liotta, N. Sandulescu, T. Vertse, Phys. Rev. Lett. 89 (2002) 042501.

[6] R. Id Betan, R.J. Liotta, N. Sandulescu, T. Vertse, Phys. Rev. C 67 (2003) 014322.

[7] N. Michel, W. Nazarewicz, M. Ploszajczak, K. Bennaceur, Phys. Rev. Lett. 89 (2002) 042502.

[8] N. Michel, W. Nazarewicz, M. Ploszajczak, J. Okolowicz, Phys. Rev. C 67 (2003) 054311.

[9] R.J. Liotta, E. Maglione, N. Sandulescu, T. Vertse, Phys. Lett. B 367 (1996) 1.

[10] T. Berggren, P. Lind, Phys. Rev. C 47 (1993) 768.

[11] A.B. Migdal, A.M. Perelomov, V.S. Popov, Yad. Fiz. 14 (1971) 874 (in Russian), Sov. J. Nucl. Phys 14 (1972) 488.

[12] I.J. Thompson, M.V. Zhukov, Phys. Rev. C 49 (1994) 1904.

[13] Y. Suzuki, R.G. Lovas, K. Yabana, K. Varga, Structure and Reactions of Light Exotic Nuclei, Taylor and Francis, London, 2003.

[14] H.G. Bohlen, et al., Nucl. Phys. A 616 (1997) 254c.

[15] G.F. Bertsch, H. Esbensen, Ann. Phys. 209 (1991) 327.

[16] J.C. Pacheco, N. Vinh Mau, Phys. Rev. C 65 (2002) 044004.

[17] H. Esbensen, G.F. Bertsch, K. Hencken, Phys. Rev. C 56 (1997) 3054, and references therein.

[18] F. Barranco, et al., Eur. Phys. J. A 11 (2001) 385

[19] K. Varga, Y. Suzuki, R.G. Lovas, Phys. Rev. C 66 (2002) 041302(R).

[20] A. Bianchini, R.J. Liotta, N. Sandulescu, Phys. Rev. C 63 (2001) 024610.

[21] E. Garrido, D.V. Fedorov, A.S. Jensen, Nucl. Phys. A 700 (2002) 117.

[22] M.V. Zhukov, L.V. Chulkov, D.V. Fedorov, D.V. Danilin, J.M. Bang, J.S. Vaagen, I.J. Thompson, J. Phys. G 20 (1994) 201.

[23] A. Cobis, D.V. Fedorov, A.S. Jensen, Phys. Lett. B 424 (1998) 1.

[24] I.J. Thomson, et al., J. Phys. G 24 (1998) 1505. 